\title{
Encuesta nacional online aplicada en pacientes con enfermedad celíaca en Chile
}

\author{
ALBERTO ESPINO ${ }^{1}$, CECILIA CASTILLO L. ${ }^{2}$, ERNESTO GUIRALDES ${ }^{3}$, \\ HELGA SANTIBÁÑEZ ${ }^{4, a}$, JUAN FRANCISCO MIQUEL ${ }^{1}$
}

\section{A national online survey applied to patients with celiac disease in Chile}

Background: Celiac disease (CD) is an immune-mediated enteropathy triggered by the ingestion of gluten in genetically susceptible individuals. Its prevalence in Europe and the USA is 0.5 to 1\%. Aim: To analyze epidemiological aspects and degree of compliance with gluten-free diet (GFD) among Chilean individuals with $C D$. Material and Methods: Subjects with confirmed or suspected CD were invited to answer an online survey published on the web at www. fundacionconvivir.cl. The answers were reinforced with a telephone interview. Results: The survey was answered by 1212 subjects (79\% females). Median age at diagnosis was 25.8 years (range 1 to 84 years), with a bimodal curve with two peaks at less than 3 years and at 20 to 40 years of age. The diagnosis was made only by serologic markers in 9\%, only by intestinal biopsy in $17.5 \%$, and by a combination of both methods in $70 \%$. Conditions associated with CD were reported by 30\% of subjects and $20 \%$ had relatives with CD. The GFD was strictly adhered to by $70 \%$, occasionally by $27 \%$ and never by $3 \%$. Seventy five percent of subjects with a strict adherence to GFD had a favorable clinical response compared with $42 \%$ of those with incomplete or lack of adherence (odds ratio 4.0, 95\% confidence intervals 2.8-5.7 p < 0.01). Conclusions: In 30\% of respondents, the diagnosis of $C D$ was not confirmed according to international guidelines that require serology and duodenal biopsy. One third of subjects recognized a poor compliance with GFD. Those with a strict adherence to it had a more favorable clinical course. However, 25\% did not experience a clinical improvement despite a strict GFD, a finding which requires further study.

(Rev Med Chile 2011; 139: 841-847).

Key words: Biopsy; Celiac disease; Diet, gluten free.

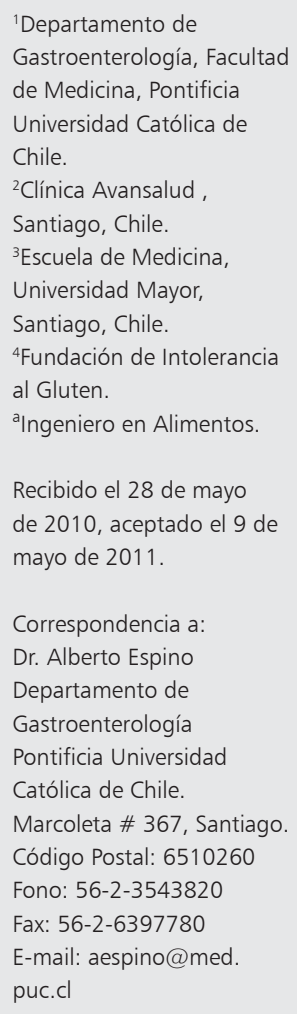

Correspondencia a: Dr. Alberto Espino Departamento de Gastroenterología Pontificia Universidad Católica de Chile. Marcoleta \# 367, Santiago. Código Postal: 6510260 Fono: 56-2-3543820 Fax: 56-2-6397780 E-mail: aespino@med. puc.cl

L a enfermedad celíaca (EC) es una enteropatía inmuno-mediada por la ingesta de gluten en personas genéticamente susceptibles. El gluten es una proteína presente en el trigo, cebada y centeno. Los principales genes predisponentes son los alelos DQ2 y DQ8 del antígeno de leucocitos de histocompatibilidad (HLA), que están presentes en al menos 95\% de los pacientes. La EC fue considerada en un principio como un raro síndrome de mala absorción y trastorno del desarrollo en la infancia, pero actualmente se considera como una enfermedad común que puede afectar a muchos otros órganos y sistemas (hígado, huesos, piel, sistema nervioso, corazón, etc. $)^{1-5}$.

La EC es común en todo el mundo y afecta entre $0,5-1 \%$ de la población general en Europa y EE.UU. de Norteamérica. Puede presentarse en cualquier grupo etario, desde infantes hasta ancianos. Su diagnóstico es más frecuente en los tres primeros años de vida después del destete y en la cuarta década de la vida, siendo más frecuente en mujeres. El retraso en el diagnóstico es de 4,5 a 9 años después del comienzo de los síntomas. Habitualmente, por cada paciente adulto diagnosticado 
de EC, se estiman ocho casos no detectados ${ }^{6-12}$. En numerosos pacientes los síntomas son interpretados durante largo tiempo como síndrome de intestino irritable ${ }^{13}$. En la actualidad, la certificación diagnóstica de EC en la gran mayoría de los casos se fundamenta en la presencia de marcadores serológicos específicos positivos, siendo los más usados los anticuerpos anti-transglutaminasa tisular (tTG) y/o anti-endomisio (EMA), seguido de un estudio histológico de la mucosa duodenal tomada mediante endoscopia ${ }^{5,6}$.

La piedra angular del tratamiento de la EC es la dieta libre de gluten (DLG). Por esta razón el consejo nutricional experto es fundamental. La DLG debe mejorar los síntomas y prevenir los déficits nutricionales y complicaciones relacionados a la EC. Por lo tanto, debe abarcar la educación respecto al suplemento de hierro, ácido fólico, vitamina B-12, fibra, calcio y vitamina $\mathrm{D}^{14}$. La adherencia a la DLG puede verse afectada por múltiples variables como la falta de educación sobre la enfermedad, el mayor costo y las dificultades de acceso de canastas libres de gluten (LG), el limitado apoyo médico y nutricional, o la ausencia de síntomas significativos que motiven a llevar una DLG de por vida ${ }^{15}$. Por esto, es relevante evaluar el grado de adherencia en forma periódica (al menos $1 \mathrm{vez}$ al año) y adoptar las medidas correspondientes para corregirla.

En Chile desconocemos aún la prevalencia poblacional de EC, siendo escasos los estudios en cuanto a sus aspectos epidemiológicos, pero el incremento de la tasa de diagnóstico en el último tiempo sugiere un considerable número de pacientes históricamente subdiagnosticados, similar a la experiencia de otros países ${ }^{16,17}$.

Los objetivos del presente estudio son analizar aspectos epidemiológicos descriptivos, y la adherencia a una DLG en una población de sujetos celíacos chilenos inscritos sistemáticamente en Fundación Convivir.

\section{Materiales y Métodos}

Estudio basado en auto-reporte voluntario de sujetos chilenos con diagnóstico confirmado o presunto de EC, mediante una encuesta estructurada online publicada en el portal de la Fundación de Intolerancia al Gluten Convivir (www.fundacionconvivir.cl), y reforzada por encuesta telefónica realizada por voluntarios de la Fundación en el período mayo 2007 a julio 2009. La encuesta incluyó aspectos epidemiológicos, género, edad al momento del diagnóstico, datos demográficos, procedencia regional, métodos diagnósticos utilizados (serología y/o biopsia intestinal), enfermedades asociadas, antecedentes familiares de EC, frecuencia de control médico, adherencia a DLG y respuesta clínica.

La adherencia a DLG fue evaluada como: estricta, ocasional y nunca. Se consideró como adherencia estricta a DLG, cuando el encuestado refirió ingerir sólo productos certificados LG publicados por Convivir o los que indican con un sello o palabra que son LG. La adherencia ocasional, fue definida como consumo periódico de productos con gluten, consciente de haber transgredido la dieta, pudiendo experimentar o no síntomas. La frecuencia puede ser una vez al mes o a lo menos 5 veces al año. La no adherencia a $D L G$, fue definida cuando el paciente refirió consumir alimentos que contienen trigo, cebada, centeno y sus subproductos. La frecuencia es de todos los días o cada semana.

La respuesta clínica a la DLG fue evaluada como: favorable, sin cambios o desfavorable. Respuesta clínica favorable, fue definida cuando el encuestado refirió una disminución significativa en la frecuencia o intensidad de sus síntomas: dolor abdominal, distensión abdominal, diarrea, constipación, compromiso del estado general, baja de peso y/o anemia. Respuesta clínica sin cambios, fue definida cuando el encuestado no refirió cambios en la frecuencia o intensidad de sus síntomas. Respuesta clinica desfavorable, fue definida cuando el encuestado refirió un incremento en la frecuencia o intensidad de sus síntomas.

\section{Consideraciones éticas}

Este estudio fue aprobado por el Comité de Ética en Investigación de la Facultad de Medicina de la Pontificia Universidad Católica de Chile. Número de proyecto: 10-032.

\section{Estadística}

Se utilizó estadística descriptiva para analizar los aspectos epidemiológicos. Para determinar diferencias entre DLG y respuesta clínica se usó test de $\chi^{2}$. En el análisis estadístico se usó el programa SPSS 15.0 para Windows, considerándose significativo todo valor $\mathrm{p}<0,05$. 
Adherencia a la dieta en pacientes con enfermedad celíaca -A. Espino et al

\section{Resultados}

\section{Aspectos epidemiológicos y diagnósticos}

El total de sujetos encuestados fue 1.212. La edad promedio al momento del diagnóstico fue 25,8 años ( 1 a 84 años). Hubo una distribución bimodal al momento del diagnóstico de EC, con un pico antes de los 3 años de edad y el otro, entre los 20 y 40 años (Figura 1). En 70\% de los casos, la EC fue diagnosticada en la vida adulta ( $>15$ años). El $79,3 \%$ fueron mujeres. Las regiones con mayor representación absoluta fueron la Región Metropolitana (RM), la V y la VIII (Tabla 1). El diagnóstico se sustentó en marcadores serológicos positivos para EC y biopsia intestinal compatible (Tabla 2). Enfermedades asociadas con EC fueron reportadas en 30\% (Tabla 3).El 20\% de los encuestados refirió presentar al menos un familiar de primer o segundo grado con EC. La frecuencia de controles médicos reportada fue cada 3 meses $1,5 \%$, cada 6 meses 49,3\%, 1 vez al año 23,4\%, y $25,8 \%$ de los pacientes refirió no controlarse.

\section{Adherencia a DLG}

El consumo de pan LG fue "todos los días" en 31\%, "a veces" $38 \%$ y "nunca" 31\%. El 17\% de los sujetos refirió preparar pan LG en casa "siempre", el 43\% "a veces", y el 40\%, "nunca". El 90\% dice consumir productos especiales LG (galletas $78,5 \%$, harinas $73,4 \%$ y pastas $66,6 \%)$. Los sujetos reportaron adherir a una DLG estricta en el 70\% de los casos, ocasional en el 27\% y nula, en el 3\%. El reporte de cumplimiento estricto a DLG se asoció a una significativa mayor respuesta clínica favorable $(74,5 \%)$, comparado con una DLG ocasional o nula (42\%) (OR 4,0 IC 95\% 2,83-5,66 p $<0,01$ ) (Figura 2).
Tabla 1. Distribución de sujetos encuestados con EC según regiones de Chile

\begin{tabular}{|c|c|c|}
\hline $\mathbf{n}$ & Región de Chile & Frecuencia \% \\
\hline$X V$ & Arica y Parinacota & 1 \\
\hline I & Tarapacá & 3,2 \\
\hline II & Antofagasta & 2,2 \\
\hline III & Atacama & 0,6 \\
\hline IV & Coquimbo & 2,8 \\
\hline V & Valparaíso & 7,3 \\
\hline RM & Metropolitana & 63 \\
\hline VI & O'Higgins & 4,3 \\
\hline VII & Maule & 2,4 \\
\hline VIII & Biobío & 6,3 \\
\hline IX & Araucanía & 2,1 \\
\hline XIV & Los Ríos & 1 \\
\hline$x$ & Los Lagos & 2,6 \\
\hline$X I$ & Aysén & 0,3 \\
\hline XII & Magallanes & 0,9 \\
\hline
\end{tabular}

Tabla 2. Métodos usados para fundamentar el diagnóstico de EC

\begin{tabular}{|lc|}
\hline Métodos diagnósticos & Frecuencia \% \\
\hline Realización de algún marcador serológico & 79 \\
Uso de anticuerpos anti-transglutaminasa (tTG) & 62 \\
Uso de anticuerpos anti-endomisio (EMA) & 59 \\
Uso de anticuerpos anti-gliadina (AGA) & 22 \\
Realización de biopsia intestinal (duodeno) & 85 \\
Diagnóstico basado sólo en serología & 9 \\
\hline Diagnóstico basado sólo en biopsia & 17,5 \\
\hline Diagnóstico basado en serología y biopsia & 70 \\
\hline Diagnóstico no basado en serología o biopsia & 3,5 \\
\hline
\end{tabular}

Tabla 3. Enfermedades asociadas con EC

\begin{tabular}{|lc|}
\hline Enfermedades asociadas & Frecuencia \% \\
\hline Hipotiroidismo & 11,8 \\
\hline Osteopenia-osteoporosis & 9,5 \\
\hline Anemia & 2,9 \\
DM tipo 1 & 1,9 \\
Cáncer digestivo & 0,3 \\
$\begin{array}{l}\text { Otras: dermatitis herpetiforme, hepatitis autoinmune, } \\
\text { colangitis esclerosante primaria (CEP), enfermedad mix- } \\
\text { ta del tejido conjuntivo (EMTC), síndrome de Sjögren, } \\
\text { CREST, epilepsia, miastenia gravis, depresión, infertilidad }\end{array}$ & \\
\hline
\end{tabular}




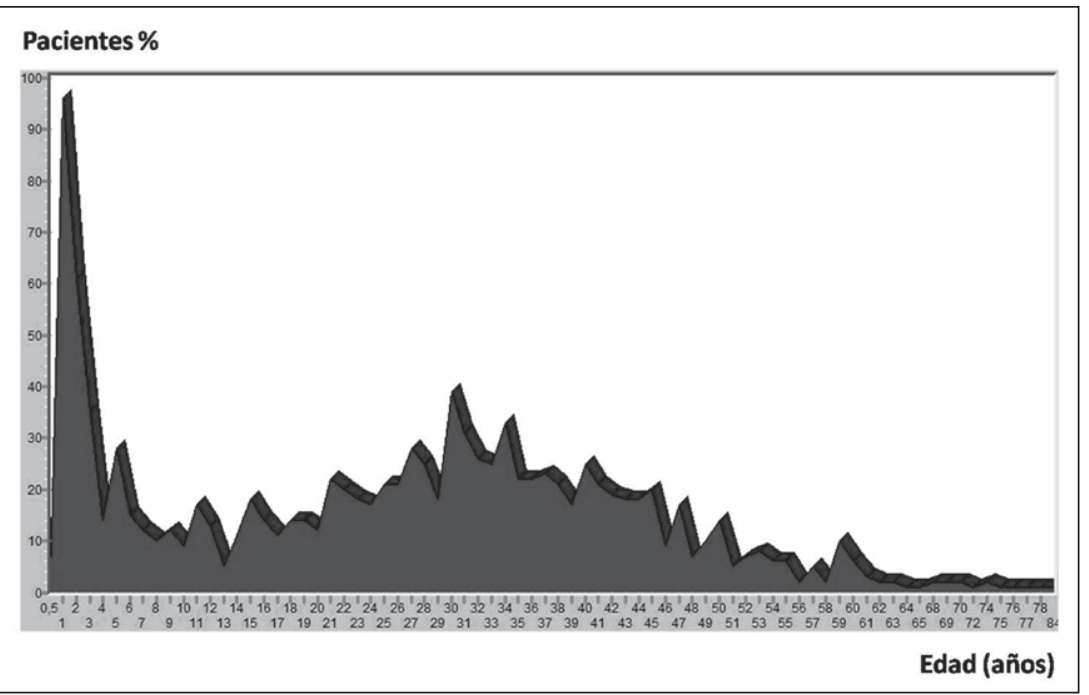

Figura 1. Distribución de pacientes con EC encuestados según edad al momento del diagnóstico.

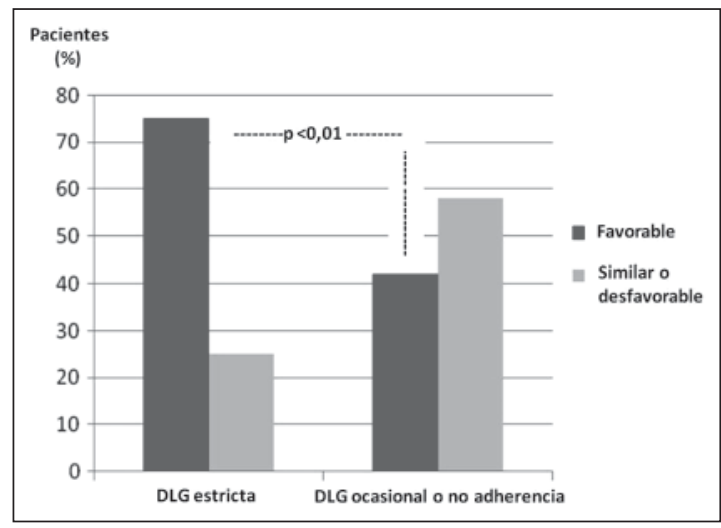

Figura 2. Relación del cumplimiento a DLG y respuesta clínica. El cumplimiento de una DLG estricta se asocia a mayor respuesta clínica favorable $(74,5 \%)$ comparado a una DLG ocasional o no adherencia (42\%) (OR 4,0, IC 95\% 2,83-5,66, $\mathrm{p}<0,01)$.

\section{Discusión}

Nuestro estudio incluyó un número considerable de pacientes con diagnóstico cierto o presunto de EC a lo largo de Chile, encontrándose representadas todas sus regiones. Las tres regiones con mayor representación en términos absolutos RM, V y VIII, coinciden con las zonas más pobladas de nuestro país. Sin embargo, cabe destacar que el porcentaje de cada región no se correlaciona proporcionalmente con la población total esti- mada de cada región. De hecho, el 63\% de los encuestados son de la RM, siendo que esta región contiene al $38 \%$ de la población chilena ${ }^{18}$. Si bien desconocemos aún cuál es la prevalencia de EC por regiones a nivel nacional, es probable que exista un menor registro voluntario en provincias que en relación a RM. Los resultados deben hacernos reflexionar sobre como aumentar la relativamente baja participación regional en esta encuesta online, incrementando la difusión del sitio web tanto a nivel de los pacientes como de los profesionales de la salud.

El presente estudio describe la experiencia de un registro sistemático y voluntario de pacientes celíacos chilenos que acceden a la Fundación de Intolerancia al Gluten, Convivir, motivados a encontrar un sitio en común donde pueden educarse en forma continua (página web, mail de consulta al experto, artículos científicos, organización de talleres y cursos), compartir sus experiencias, solucionar dudas y problemas asociados con la DLG, la certificación de productos LG e inquietudes referentes a la misma. Debemos destacar que en Chile existe otra importante Corporación de Apoyo al Celíaco, COACEL (www.coacel.cl), a la cual los pacientes pueden acceder para obtener información y apoyo.

Los resultados obtenidos tienen el valor y las limitaciones propias de un registro voluntario, realizado vía web y reforzado vía telefónica por voluntarios no médicos, por lo que deben ser 
interpretados en ese contexto. Llama la atención que en 3,5\% de los encuestados, el diagnóstico de EC no había sido documentado, es decir, no se habría llevado a cabo en ellos serología o una biopsia intestinal o simplemente el encuestado no recuerda los estudios realizados. Por otra parte, en $17,5 \%$ el diagnóstico se habría basado sólo en estudio de biopsia, y en 9\% sólo con serología, sin confirmación histológica. Con escasas excepciones, el diagnóstico de EC debe sustentarse en la existencia de marcadores serológicos específicos positivos (tTG y/o EMA) más el análisis histológico de una biopsia duodenal compatible; esto se cumplió sólo en el 70\% de los casos analizados. La elevada proporción de individuos que han sido rotulados como celíacos en ausencia de una apropiada confirmación diagnóstica de acuerdo a las guías internacionales ${ }^{5,6}$ es inquietante, ya que pudiera indicar una relativa falta de rigor en la conceptualización de una condición crónica, que impone limitaciones en la calidad de vida de quienes la padecen ${ }^{19}$.

En cuanto a los aspectos epidemiológicos, destaca que la edad al momento del diagnóstico de EC fue en $70 \%$ de los casos en población adulta definida como mayores de 15 años, tendencia que coincide con lo descrito en la literatura en las últimas dos décadas. Un estudio reciente en Gran Bretaña ha demostrado que el diagnóstico de EC se efectúa actualmente con una mayor frecuencia en adultos que en niños, a una razón de 9:1 ${ }^{8}$. Es interesante considerar que en $3 \%$ de nuestros casos el diagnóstico se realizó después de los 60 años, teniendo el paciente más anciano la edad de 84 años. Estudios epidemiológicos han sugerido que un porcentaje sustancial de pacientes son diagnosticados después de los 50 años. Incluso, en un estudio, el promedio de edad al diagnóstico fue cercano a los 50 años, con un tercio de los nuevos pacientes diagnosticados cercanos a los 65 años $^{20}$.

La distribución por género fue predominante femenino a una razón de 4:1. Esta observación es también similar a lo publicado en otras series contemporáneas, donde en promedio la relación de mujeres sobre hombres es 3 veces a 1. Las razones de esto son desconocidas, pero se cree que puede obedecer a la mayor prevalencia en mujeres de enfermedades autoinmunes en general, $y$ al mayor estudio de anemia ferropriva y osteoporosis en mujeres. Esta predominancia disminuye luego de los 65 años $^{21}$.
Un tercio de los pacientes presenta, al menos, una enfermedad asociada con EC, destacando hipotiroidismo, osteopenia-osteoporosis y anemia, complicaciones frecuentes en otras poblaciones de EC. Llama la atención el 11,8\% de auto-reporte de hipotiroidismo, siendo casi el doble de lo reportado en la literatura ${ }^{8}$. Por ello, es importante buscar estas enfermedades asociadas en pacientes con EC en forma dirigida y tratarlas oportunamente, como también sospechar la existencia de EC en aquellos pacientes que son evaluados por estas condiciones médicas de prevalencia relativamente frecuente.

El segundo objetivo de este estudio fue analizar la adherencia a una DLG y su respuesta clínica. Un tercio de quienes respondieron la encuesta reconoce mala adherencia a DLG. La adherencia estricta a DLG se asoció con respuesta clínica favorable, disminución significativa de la frecuencia e intensidad de síntomas clínicos. Sin embargo, 25\% de los celíacos encuestados refirió no experimentar mejoría clínica a pesar de llevar una estricta DLG, lo que requeriría mayor estudio. Se podría especular que en este subgrupo de pacientes podría existir ingesta inadvertida de gluten (por contaminación cruzada o por desconocimiento) y/o comorbilidad con trastornos funcionales gastrointestinales (ej. síndrome de intestino irritable o mala absorción a lactosa primaria del adulto), los cuales son de alta frecuencia en la población general. También, es posible que en este subgrupo esté presente una sustancial proporción de los individuos autorrotulados como celíacos en esta serie, que no tienen una adecuada certificación diagnóstica y que es posible no sean celíacos. En ellos, obviamente, puede anticiparse que sus síntomas no van a remitir ni atenuarse con DLG, siendo posible también que una elevada proporción de ellos presente básicamente un trastorno digestivo funcional. Ahora, al comparar el grado de adherencia estricta a DLG del presente grupo de estudio (70\%), con otras poblaciones estudiadas y considerando todos los sesgos mencionados, nos situaríamos dentro de una realidad similar a lo descrito en poblaciones de otros países, cuyo rango de adherencia a DLG fluctúa entre $42 \%$ al $91 \%{ }^{22}$.

La mayor limitante por la naturaleza del presente estudio, es el sesgo de selección basado en un sesgo de reporte, en este caso, auto-reporte. No tenemos constancia en muchos casos de que el diagnóstico se haya hecho rigurosamente y que el 
tratamiento sea el adecuado, o que el paciente comunique fehacientemente lo que se le ha efectuado desde un punto de vista médico. Es posible, por ejemplo, que los que se inscriban en la encuesta de Convivir sean aquéllos que tienen más síntomas, o los que presentan más dudas o, por último, los que tienen acceso a un computador y capacidades para interactuar con un sitio web e iniciativa para educarse y tratarse.

Por otro lado, la fortaleza de este estudio está a nuestro juicio en el gran número de encuestados, siendo la serie más grande comunicada en Chile hasta el momento, otorgando datos que nos muestran "una realidad subjetiva" de la enfermedad a lo largo de todo el país, sugiriendo que la EC es un importante problema de salud pública. En Chile tenemos muchos desafíos pendientes en esta área, desde conocer su prevalencia poblacional real, el desarrollo de programas de pesquisa y diagnóstico precoz a nivel primario y secundario, como también una mejor estructuración de apoyo terapéutico y certificación de alimentos LG. Un mejor conocimiento de la epidemiología de la EC a nivel nacional, nos permitirá avanzar en regularizar normativas legales respecto a la rotulación de alimentos LG por parte de las empresas del rubro, tal como lo sugieren los países desarrollados ${ }^{5,6}$.

En suma, los enfermos auto-reportados con EC a lo largo de Chile presentan una distribución por sexo y edad similares a lo descrito en otras poblaciones. En el 30\% de los casos la certificación diagnóstica no pareció seguir las recomendaciones internacionales de serología y biopsia. Un tercio reconoce mala adherencia a DLG. La adherencia estricta a DLG se asocia una respuesta clínica favorable. Sin embargo, 25\% refiere no experimentar mejoría clínica a pesar de cumplir estrictamente con la DLG, lo que requeriría mayor estudio.

Agradecimientos. Queremos agradecer la fundamental iniciativa de los miembros de Fundación Convivir en el diseño y desarrollo de este estudio y por su desinteresada labor en la educación y promoción de la EC en todo Chile, especialmente a Chantal Signorio y Carolina Marambio.

\section{Referencias}

1. Tack G, Verbeek, Schureurs M, Mulder C. The spectrum of celiac disease: epidemiology, clinical aspects and treatment. Nature Reviews Gastroenterology \& Hepatology 2010; 7: 204-13.

2. Catassi C, Fasano A. Celiac disease. Current Opinion in Gastroenterology 2008; 24: 687-91.

3. Wolters VM, Wijmenga C. Genetic background of celiac disease and its clinical implications. Am J Gastroenterol 2008; 103: 190-5.

4. Green PHR, Cellier C. Celiac Disease. N Engl J Med 2007; 357: 1731-43.

5. Rostom A, Murray JA, Kagnoff MF. American Gastroenterological Association Technical Review (AGA) Institute on the Diagnosis and Management of Celiac Disease. Gastroenterology 2006; 131: 1981-2002.

6. Troncone R, Ivarsson A, Szajewska H, Mearin ML. Review article: future research on coeliac disease a position report from the European multistakeholder platform on celiac disease (CDEUSSA). Aliment Pharmacol Ther 2008; 27: 1030-43.

7. Hopper A, Hadjivassiliou M, Butt S, Sanders D. Adult coeliac disease. BMJ 2007; 335: 558-62.

8. West J, Logan Rf, Hill Pg, Lloyd A, Lewis S, Hubbar R, et al. Seroprevalence, correlates, and characteristics of undetected coeliac disease in England. Gut 2003; 52: 960-5.

9. Maki M, Mustalahti K, Kokkononen J, Kulmala P, Haapalahti M, Karttunen T, et al. Prevalence of celiac disease among children in Finland. N Engl J Med 2003; 348: 2517-24.

10. Tatar G, Elsurer R, Simsek H, Balaban YH, Hascelik G, Buyukasik Y, et al. Screening of tissue transglutaminase antibody in healthy blood donors for celiac disease screening in the Turkish population. Dig Dis Sci 2004; 49: 1479-84.

11. Fasano A, Berti I, Gerarduzzi T, Not T, Colletti RB, Drago $S$, et al. Prevalence of celiac disease in at-risk and notat-risk groups in the United States: a large multicenter study. Arch Intern Med 2003; 163: 286-92.

12. Bingley PJ, Williams AJ, Norcross AJ, Unsworth DJ, Lock RJ, Ness AR, et al. Undiagnosed coeliac disease at age seven: population based prospective birth cohort study. BMJ 2004; 328: 322-3.

13. Ford AC, Chey WD, Talley NJ, Malhotra A, Spiegel BM, Moayyedi P. Yield of Diagnostic Tests for Celiac Disease in Individuals with Symptoms Suggestive of Irritable Bowel Syndrome: Systematic review and meta-analysis. Arch Intern Med 2009; 169: 651-8.

14. Thompson T, Dennis M, Higgins LA, Lee AR, Sharrett MK. Gluten-free diet survey: are Americans with coeliac disease consuming recommended amounts of fibre, iron, calcium and grain foods? J Hum Nutr Diet 2005; 18: 163-9. 
Adherencia a la dieta en pacientes con enfermedad celíaca -A. Espino et al

15. Ciacci C, Cirillo M, Cavallaro R, Mazzacca G. Long-term follow-up of celiac adults on gluten-free diet: prevalence and correlates of intestinal damage. Digestion 2002; 66: 178-85.

16. Mancilla C, Madrid AM, Valenzuela J, Morales A, Hurtado C, Smok G, et al. Enfermedad celíaca del adulto: Experiencia clínica. Rev Med Chile 2005; 133: 1317-21.

17. Canales P, Araya M, Alliende F, Hunter B, Alarcón T, Chávez E. Estado actual del diagnóstico y presentaciones clínicas de enfermedad celíaca. Estudio multicéntrico. Rev Med Chile 2008; 136: 296-303.

18. Censo de Población Chilena 2002. Disponible en: www. ine.cl.
19. Whitaker JK, West J, Holmes GK, Logan RF. Patient perceptions of the burden of coeliac disease and its treatment in the UK. Aliment Pharmacol Ther 2009; 29: 1131-6.

20. Rashtak S, Murray JA. Celiac disease in the elderly. Gastroenterol Clin North Am 2009; 38: 433-46.

21. Green PHR, Stavropoulos SN, Panagi SG, Goldstein SL, Mcmahon DJ, Absan H, et al. Characteristics of adult celiac disease in the USA: results of a national survey. Am J Gastroenterol 2001; 96: 126-31.

22. Hall NJ, Rubin G, Charnock A. Systematic review: adherence to a gluten-free diet in adult patients with coeliac disease. Aliment Pharmacol Ther 2009; 30: 315-30. 